\title{
Vector analysis of the current commutation in PM BLDC drives
}

\author{
G. BUJA ${ }^{1 *}$, R. MENIS ${ }^{2}$, and R. KESHRI ${ }^{1}$ \\ ${ }^{1}$ Department of Industrial Engineering, University of Padova, 6/a Gradenigo St., 35131 Padova, Italy \\ ${ }^{2}$ Department of Engineering and Architecture, University of Trieste, 10 A. Valerio St., 34127 Trieste, Italy
}

\begin{abstract}
The vector approach in either stationary or synchronous plane is commonly used for the analysis of the AC drives like induction and PM brushless AC drives whilst it has been hardly ever used for the PM brushless DC (BLDC) drives. A possible reason is that they require injection of square-wave currents into the motor phases, which are non-sinusoidal in nature. Recently, the PM BLDC drives have been analyzed by the help of the vector approach in the stationary plane. This paper applies such an approach to an in-depth analysis of the operation of the PM BLDC drives during the current commutations, illustrating the potentialities of the approach in giving a better insight into the current transients and the ensuing torque characteristics. At last, two voltage control strategies proposed to eliminate the torque ripple due to the current commutations are considered, showing that they can be readily understood by the vector approach.
\end{abstract}

Key words: PM BLDC drive; current commutation; stationary plane vector analysis.

\section{Introduction}

PM BLDC drives are characterized by the capability of offering higher torque and power density compared to other $\mathrm{AC}$ drives of the same size. These features together with compact structure and cost effectiveness of the motor have made the PM BLDC drives widely accepted for motorizing many industrial equipment [1-3] and, nowadays, for propelling electric vehicles [4-6]. PM BLDC drives require injection of squarewave currents into the motor phases, synchronized with the flat portion of the trapezoidal back-emf to develop a constant torque and -at the same time- to maximize the torque-perampere ratio. As the flat portion of the back-emf covers $120^{\circ}$ electrical angle, the voltage source inverter (VSI) supplying the motor uses its $120^{\circ}$ working mode to inject square-wave currents into the motor phases. Commutation of the phase currents takes place with an electrical angle periodicity of $60^{\circ}$ and should be instantaneous [7]. In practice, phase inductances and limited supply voltage yield a finite time for the commutations and the currents deviate from the required waveform. Any mismatch in the current waveform impairs the drive performance as it produces: i) a drop of the speed-torque characteristic at high speeds that limits the full utilization of the drive in all the speed range, and ii) the onset of torque ripples over almost all the speed range that give rise to an uneven motion of the attached mechanical load and an unduly solicitation on the rotor bearings [8]. Here, torque ripples designate the torque pulsations due to the current commutations. Other types of torque pulsations exist; they are due to the current oscillations originated by the PWM control of the VSI and to the cogging phenomena originated by the interaction between the permanent magnets of the rotor and the slots of the stator. Torque pulsations due to the current oscillations occur at high frequencies so that their impact on the load speed is very much attenuated by the filtering action of the motor-load inertia whilst torque pulsations due to the cogging phenomena are minimized with a suitable electromagnetic design of the motor [9].

Current transients in the PM BLDC drives during the commutations have been explained in [10] for the defined low and high speed zones. The profiles of the torque developed by the motor during the commutations have been derived in [11] as a function of the motor speed by correlating currents, speed and torque of the motor. All the significant literature on the matter has approached the analysis of the PM BLDC drives by means of the phase variables. Recently, this analysis has been executed by means of the vector representation of the drive variables in the stationary plane [12].

This paper applies the vector approach to an in-depth analysis of the operation of the PM BLDC drives during the current commutations. Organization of the paper is as follows: Sec. 2 reviews the vector representation of the PM BLDC drive variables in the stationary plane; Secs. 3 and 4 exploit the vector approach to illustrate the current transients during the commutations and the ensuing torque characteristics at low and high speeds, respectively; Sec. 5 shows the potentialities of the vector approach by using it to explain the rationale behind two voltage control strategies proposed in the literature to eliminate the torque ripple due to the current commutations $[13,14]$; Sec. 6 concludes the paper.

\section{PM BLDC drive}

2.1. Operation. Circuital scheme of a conventional PM BLDC drive is shown in Fig. 1. The DC link of the drive is powered by the DC voltage source $V_{d}$ and feeds the PM BLDC motor through the VSI.

\footnotetext{
*e-mail: giuseppe.buja@unipd.it
} 


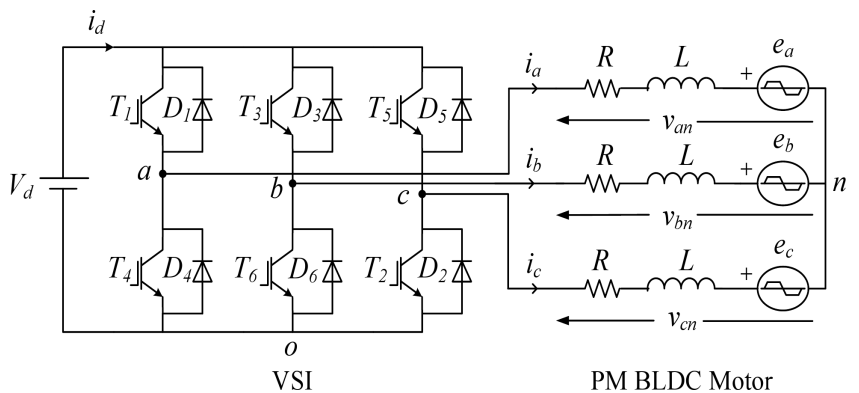

Fig. 1. Circuital scheme of a PM BLDC drive

Voltage equations for the motor phases are

$$
\begin{gathered}
\frac{d i_{j}\left(\theta_{e}\right)}{d \theta_{e}}=-\frac{R}{\Omega_{e} L} i_{j}\left(\theta_{e}\right) \\
+\frac{1}{\Omega_{e} L} \underbrace{\left[v_{j n}\left(\theta_{e}\right)-e_{j}\left(\theta_{e}\right)\right]}_{v_{j L}\left(\theta_{e}\right)} \quad j=a, b, c,
\end{gathered}
$$

where $j$ is a generic motor phase, $i_{j}, v_{j n}, v_{j L}$ and $e_{j}$ are the current flowing into the motor phases, the voltage applied to the motor phases, the voltage drop across the phase inductances, and the back-emf of the motor phases, respectively, $R$ and $L$ are the resistance and the mutual-effect inclusive inductance of the motor phases, $\theta_{e}=\Omega_{e} t$ is the angular phase in electrical radians, $\Omega_{e}=n_{p} \Omega$ is the motor speed in electrical radians per second, $n_{p}$ is the number of pole pairs, and $\Omega$ is the motor speed.

The phase voltages $v_{j n}$ are related to the VSI output voltages $v_{j o}$, measured with respect to the negative rail of the DC link, by

$$
v_{j o}=v_{j n}+v_{n o}
$$

and the phase currents are constrained by the Kirchhoff's current law

$$
\sum_{j=a, b, c} i_{j}=0
$$

The instantaneous electrical power $p$ converted into mechanical form and the corresponding motor torque $\tau$ can be expressed as

$$
\begin{gathered}
p\left(\theta_{e}\right)=\sum_{j=a, b, c} e_{j}\left(\theta_{e}\right) i_{j}\left(\theta_{e}\right), \\
\tau\left(\theta_{e}\right)=\frac{p\left(\theta_{e}\right)}{\Omega} .
\end{gathered}
$$

Operation of a PM BLDC drive is expounded with the help of Fig. 2. Back-emfs are trapezoidal with the magnitude of the flat portions equal to $+E$ or $-E$ and their duration equal to $2 \pi / 3$ (electrical) radians. The value of $E$ is given by

$$
E=k \Omega
$$

where $k$ is the motor constant. The phase currents have a square waveform with pulses of magnitude $+I$ or $-I$ that are synchronized with the flat portions of the back-emfs. For motor operation, the phase currents in Fig. 2 have the same sign as the polarity of the back-emfs.

From Fig. 2, it emerges that the supply period of the motor can be divided into six supply intervals $S_{1}, S_{2}, \ldots$, and $S_{6}$ of the duration of $\pi / 3$ radians. At the beginning of each supply interval, one phase starts to conduct (incoming phase) and another one finishes of conducting (outgoing phase). During the remaining part of the supply interval, only two-phases with back-emfs of opposite polarities conduct (conducting phases). To identify the supply interval and, from it, the conducting transistors of the VSI, the drive control uses the information delivered by three Hall sensors mounted on the stator and displaced of $2 \pi / 3$ electrical radians. The required voltage is applied to the conducting phases by stepping down the DC link voltage $V_{d}$ through the choppering of the conducting transistors.

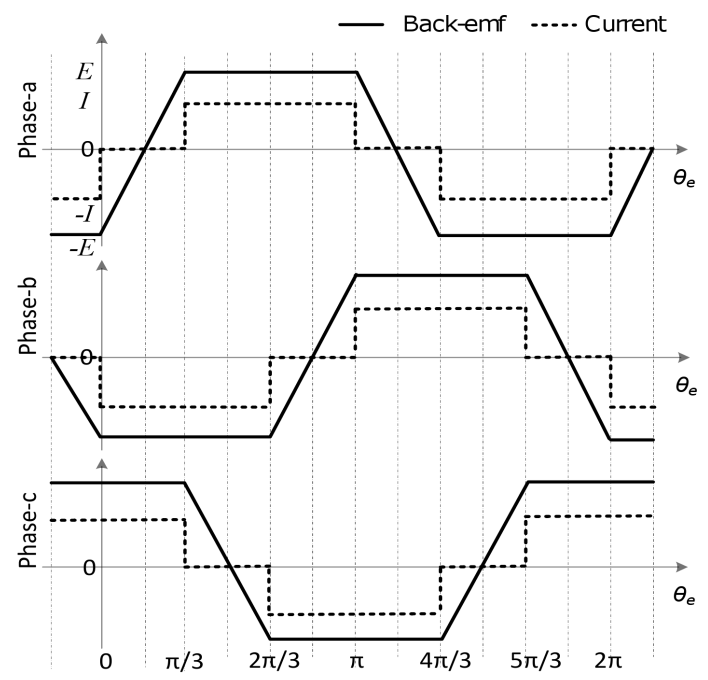

$$
\begin{aligned}
& \begin{array}{cccccccccc}
\text { Supply } & \mathrm{S}_{6} & \mathrm{~S}_{1} & \mathrm{~S}_{2} & \mathrm{~S}_{3} & \mathrm{~S}_{4} & \mathrm{~S}_{5} & \mathrm{~S}_{6} & \mathrm{~S}_{1}
\end{array}
\end{aligned}
$$

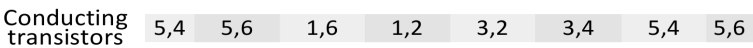

Fig. 2. Back-emfs, currents and supply intervals of a PM BLDC drive

2.2. Control system. As Fig. 3 shows, regulation of the phase currents at the reference value $I_{\text {ref }}$ can be accomplished by the closed-loop control of the DC link current $i_{d}$. In fact, this current remains equal to the current flowing into a motor phase when both the conducting transistors are ON; therefore, if properly sampled, the DC link current gives the feedback of the phase currents. The current error is processed by a PI regulator that delivers the required voltage $v_{c}$ for the motor phases.

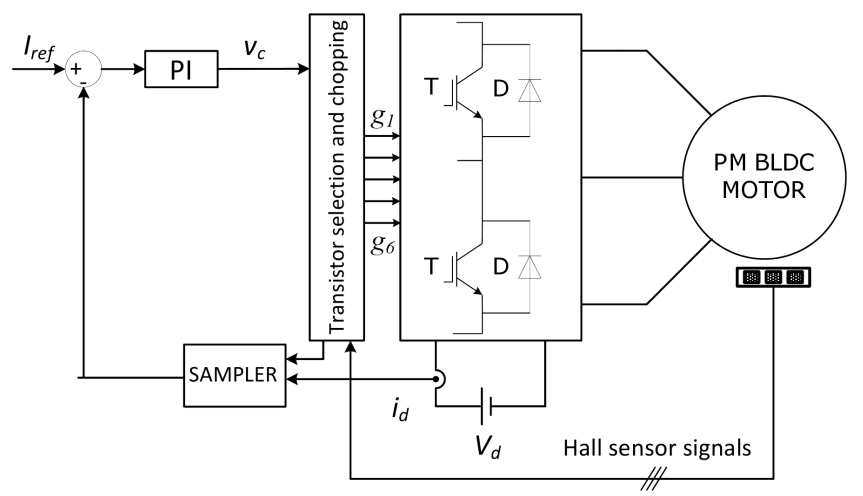

Fig. 3. Schematic of the control of a PM BLDC drive 
Application of $v_{c}$ across the phases of the motor is commonly attained by chopping only one of the two conducting transistors to reduce the switching losses; a common strategy is to chop the transistor carrying either the incoming or the outgoing current. Hereafter, it is assumed that the control applies the required voltage without any delay.

2.3. Stationary plane representation. Equations of the PM BLDC motor can be written in terms of the vectors $i=$ $\left[i_{\alpha} i_{\beta}\right]^{T}, \boldsymbol{e}=\left[e_{\alpha} e_{\beta}\right]^{T}$, and $\boldsymbol{v}=\left[v_{\alpha} v_{\beta}\right]^{T}$ of the currents and the back-emfs of the motor phases, and the VSI output voltages in the stationary plane. The vectors, calculated with a magnitude-invariant transformation, are given by

$$
\begin{aligned}
\boldsymbol{i}\left(\theta_{e}\right) & =i_{a}\left(\theta_{e}\right)+j \frac{1}{\sqrt{3}}\left[i_{b}\left(\theta_{e}\right)-i_{c}\left(\theta_{e}\right)\right], \\
\boldsymbol{v}\left(\theta_{e}\right)= & \frac{2}{3}\left[v_{a o}\left(\theta_{e}\right)-\frac{v_{b o}\left(\theta_{e}\right)}{2}-\frac{v_{c o}\left(\theta_{e}\right)}{2}\right] \\
& +j \frac{1}{\sqrt{3}}\left[v_{b o}\left(\theta_{e}\right)-v_{c o}\left(\theta_{e}\right)\right], \\
e\left(\theta_{e}\right)= & \frac{2}{3}\left[e_{a}\left(\theta_{e}\right)-\frac{e_{b}\left(\theta_{e}\right)}{2}-\frac{e_{c}\left(\theta_{e}\right)}{2}\right] \\
& +j \frac{1}{\sqrt{3}}\left[e_{b}\left(\theta_{e}\right)-e_{c}\left(\theta_{e}\right)\right] .
\end{aligned}
$$

By (7), the voltage equations in (1) become

$$
\frac{d \boldsymbol{i}\left(\theta_{e}\right)}{d \theta_{e}}=-\frac{R}{\Omega_{e} L} \boldsymbol{i}\left(\theta_{e}\right)+\frac{1}{\Omega_{e} L} \underbrace{\left[\boldsymbol{v}\left(\theta_{e}\right)-\boldsymbol{e}\left(\theta_{e}\right)\right]}_{\boldsymbol{v}_{L}\left(\theta_{e}\right)}
$$

Calculation of the current and back-emf vectors for the waveforms in Fig. 2 shows that: i) the current vector $i$ remains stationary within the supply intervals $S_{1}, S_{2}, \ldots$, and $S_{6}$, and coincides with the radii $\boldsymbol{I}_{1}, \boldsymbol{I}_{2}, \ldots$, and $\boldsymbol{I}_{6}$, respectively, of an hexagon (current hexagon), ii) the back-emf vector $\boldsymbol{e}$ moves along the sides of an hexagon (back-emf hexagon) during the supply intervals, and coincides with the radii $\boldsymbol{E}_{1}, \boldsymbol{E}_{2}, \ldots$, and $\boldsymbol{E}_{6}$ of the hexagon at the beginning of the supply intervals. Current and back-emf vectors are traced in Fig. 4 whilst their values as a fraction of $I$ and $E$ are reported in Table 1 .

As an example, in the supply interval $S_{1}$ the current vector jumps from $\boldsymbol{I}_{6}(-I, I / \sqrt{ } 3)$ to $\boldsymbol{I}_{1}(0,-2 I / \sqrt{ } 3)$ at $\theta_{e}=0$ and, after that, stays at $\boldsymbol{I}_{1}$ within the whole $S_{1}$. Instead, the back-emf vector takes the value $\boldsymbol{E}_{1}(-2 E / 3,-2 E / \sqrt{ } 3)$ at $\theta_{e}=0$ and moves towards $\boldsymbol{E}_{2}(2 E / 3,-2 E / \sqrt{ } 3)$ during $S_{1}$, reaching $\boldsymbol{E}_{2}$ at $\theta_{e}=\pi / 3$. For given values of $I$ and $E$, the magnitude of the current vector remains the same and equal to $2 I / \sqrt{ } 3$, whereas the magnitude of the back-emf vector undergoes a continuous change from the maximum value of $4 E / 3$ at the beginning of the supply interval to the minimum value of $2 E / \sqrt{ } 3$ at the mid of supply interval and then again to the maximum value of $4 E / 3$ at the end of the supply interval.
Table 1

Current and back-emf vectors

\begin{tabular}{ccc}
\hline \hline Supply Interval & $\begin{array}{c}\text { Current vectors } \\
\text { as a fraction of } I\end{array}$ & $\begin{array}{c}\text { Back-emf vectors } \\
\text { as a fraction of } E\end{array}$ \\
\hline$S_{1}$ & $\mathbf{I}_{\mathbf{1}}=-j \frac{2}{\sqrt{3}}$ & $\mathbf{E}_{\mathbf{1}}=-\frac{2}{3}-j \frac{2}{\sqrt{3}}$ \\
\hline$S_{2}$ & $\mathbf{I}_{\mathbf{2}}=1-j \frac{1}{\sqrt{3}}$ & $\mathbf{E}_{\mathbf{2}}=\frac{2}{3}-j \frac{2}{\sqrt{3}}$ \\
\hline$S_{3}$ & $\mathbf{I}_{\mathbf{3}}=1+j \frac{1}{\sqrt{3}}$ & $\mathbf{E}_{\mathbf{3}}=\frac{4}{3}$ \\
\hline$S_{4}$ & $\mathbf{I}_{\mathbf{4}}=j \frac{2}{\sqrt{3}}$ & $\mathbf{E}_{\mathbf{4}}=\frac{2}{3}+j \frac{2}{\sqrt{3}}$ \\
\hline$S_{5}$ & $\mathbf{I}_{\mathbf{5}}=-1+j \frac{1}{\sqrt{3}}$ & $\mathbf{E}_{\mathbf{5}}=-\frac{2}{3}+j \frac{2}{\sqrt{3}}$ \\
\hline$S_{6}$ & $\mathbf{I}_{\mathbf{6}}=-1-j \frac{1}{\sqrt{3}}$ & $\mathbf{E}_{\mathbf{6}}=-\frac{4}{3}$ \\
\hline
\end{tabular}

Power equation in (4) can be expressed in terms of the inner product of the vectors $e$ and $i$ as

$$
\begin{gathered}
p\left(\theta_{e}\right)=\frac{3}{2} \boldsymbol{e}\left(\theta_{e}\right) \cdot \boldsymbol{i}\left(\theta_{e}\right) \\
=\frac{3}{2}\left[e_{\alpha}\left(\theta_{e}\right) i_{\alpha}\left(\theta_{e}\right)+e_{\beta}\left(\theta_{e}\right) i_{\beta}\left(\theta_{e}\right)\right] .
\end{gathered}
$$

For the ideal case of instantaneous commutation, during the interval $0 \leq \theta_{e} \leq \pi / 3$ the magnitude of the projection of $e$ over $i$ is constant and equal to $2 E / \sqrt{ } 3$. Thus the motor torque in (5) turns out to be constant and equal to

$$
\mathrm{T}=2 k I \text {. }
$$

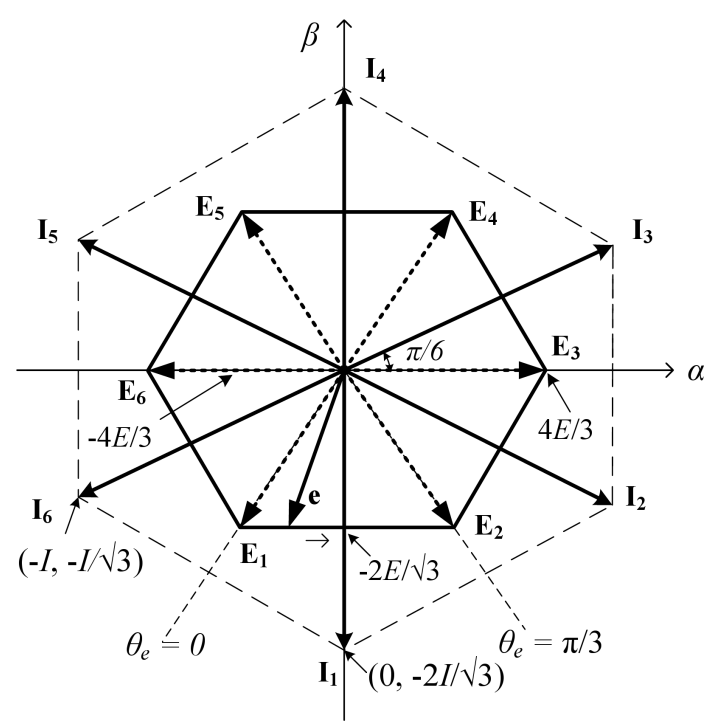

Fig. 4. Current and back-emf vectors in the stationary plane

\section{Current commutation}

In practice, the incoming and outgoing currents take some time to get the required magnitude and the current commutation is not instantaneous. The analysis of the PM BLDC drive operation during the current commutations is carried out by supposing that 
1. the voltage drop across the phase resistances is negligible compared to the other voltage terms in (8), so that it can be disregarded,

2 . the commutation interval is small compared to the maximum allowed interval of $\pi / 3$ [9] so that $e$ remains equal to the back-emf vector taken at the beginning of the relevant supply interval.

Above assumptions reduce (8) to

$$
\frac{d \boldsymbol{i}\left(\theta_{e}\right)}{d \theta_{e}}=\frac{1}{\Omega_{e} L} \underbrace{\left[\boldsymbol{v}\left(\theta_{e}\right)-\boldsymbol{E}_{\mathbf{1}}\right]}_{\boldsymbol{v}_{\boldsymbol{L}}}\left(\theta_{e}\right)
$$

for the supply interval $S_{1}$. Equation (11) shows that the change in the current vector is parallel to $\boldsymbol{v}_{L}$.
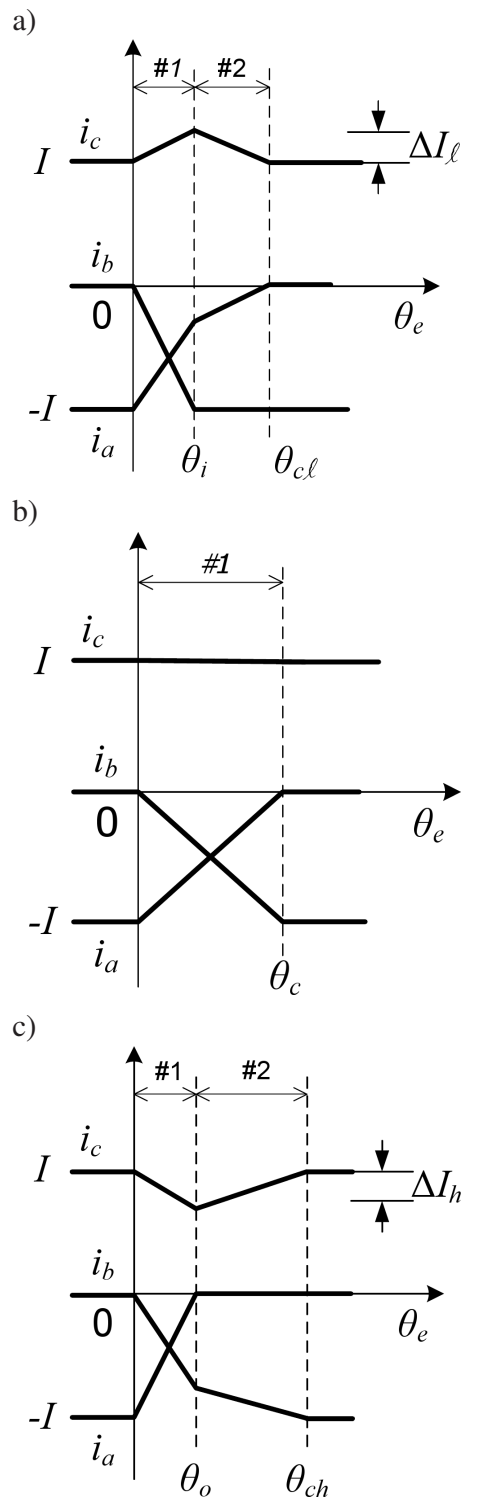

Fig. 5. Current transients during commutation: a) in the low-speed zone $\left(4 E<V_{d}\right)$, b) for $4 E=V_{d}$, and c) in the high-speed zone $\left(4 E>V_{d}\right)$

Let us consider the current commutation taking place at the beginning of the supply interval $S_{1}$, which starts at $\theta_{e}=0$ as in Fig. 2. The drive control system turns $T_{4}$ OFF and $T_{6}$ $\mathrm{ON}$, and keeps $T_{5} \mathrm{ON}$. The commutating currents are $i_{b}$ and $i_{a}$, with $i_{b}$ that is ingoing and $i_{a}$ that is outgoing, whilst $i_{c}$ is the non-commutating current. Current $i_{a}$ freewheels through $D_{1}$ until it extinguishes.

Generally, the commutation interval is divided into two subintervals, and the current transients depend on whether the motor runs in the low-speed zone, which occurs for $4 E<V_{d}$, or in the high-speed zone, which occurs for $4 E>V_{d}$ [10]. The current transients in the two speed zones and for $4 E=V_{d}$ are shown in Fig. 5, where the two commutation subintervals are marked with \#1 and \#2 and the duration of the commutation interval is denoted with $\theta_{c \ell}$ and $\theta_{c h}$, respectively. Hereafter, quantities pertinent to the low-speed zone are identified with the subscript $l$ and those to the high-speed zone with the subscript $h$.

3.1. Current transients in subinterval \#1. During subinterval \#1, all the three motor phases conduct and the VSI exerts the maximum effort to the motor for the commutating currents to reach the required magnitudes, i.e. it is $v_{a o}=V_{d}$, $v_{b o}=0$ and $v_{c o}=V_{d}$. From (7), the vector of the VSI output voltages during subinterval \#1 is

$$
\boldsymbol{V}_{1}=\left(\frac{1}{3}-j \frac{1}{\sqrt{3}}\right) V_{d}
$$

The vector has fixed magnitude and is aligned along $\boldsymbol{E}_{2}$. By (11) and (12), the vector of the voltage drops across the phase inductances is

$$
\boldsymbol{V}_{\boldsymbol{L}, 1}=\boldsymbol{V}_{\mathbf{1}}-\boldsymbol{E}_{\mathbf{1}}=\frac{1}{3}\left(V_{d}+2 E\right)-j \frac{1}{\sqrt{3}}\left(V_{d}-2 E\right) .
$$

The vector in (13) has a slope of

$$
m_{1}=-\sqrt{3}\left(\frac{V_{d}-2 E}{V_{d}+2 E}\right)
$$

which is a function of $E$ and, therefore, depends upon the motor speed $\Omega$.

Integration of (11) with the initial condition $\boldsymbol{i}(0)=\boldsymbol{I}_{6}$ leads to the current vector $i$ during subinterval \#1

$$
\boldsymbol{i}_{1}\left(\theta_{e}\right)=\frac{1}{\Omega_{e} L}\left[\frac{1}{3}\left(V_{d}+2 E\right)-j \frac{1}{\sqrt{3}}\left(V_{d}-2 E\right)\right] \theta_{e}+\mathbf{I}_{6} .
$$

From (14) and (15) it can be concluded that the current vector during subinterval \#1 moves towards $I_{1}$ along a straight line with the slope in (14). So, as shown in Fig. 6, there are three possible trajectories of the tip of the current vector: i) in the low speed zone, i.e. for $4 E<V_{d}, m_{1}$ is less than $-1 / \sqrt{ } 3$ and the tip of $\boldsymbol{i}_{1}$ moves along a line like $x$, which has an angular slope in the range from $-\pi / 6$ to $-\pi / 3$, ii) for $4 E=V_{d}, m_{1}$ becomes equal to $-1 / \sqrt{ } 3$ and the tip of $\boldsymbol{i}_{1}$ moves along the line joining $\boldsymbol{I}_{6}$ and $\boldsymbol{I}_{1}$, which has an angular slope of $-\pi / 6$, and iii) in the high speed zone, i.e. for $4 E>V_{d}, m_{1}$ becomes greater than $-1 / \sqrt{ } 3$ and the tip of $\boldsymbol{i}_{1}$ moves along a line like $w$, which has an angular slope in the range from 0 to $-\pi / 6$.

Subinterval \#1 ends in three possible modes: i) for $4 E<$ $V_{d}$, the incoming current $i_{b}$ reaches the required magnitude 
$-I$ at the angle $\theta_{i}$ before that the outgoing current $i_{a}$ vanishes (Fig. 5a); ii) for $4 E=V_{d}, i_{b}$ reaches $-I$ at the same angle that $i_{a}$ vanishes and the commutation completes at the end of the subinterval \#1, (Fig. 5b), iii) for $4 E>V_{d}, i_{a}$ vanishes at the angle $\theta_{o}$ before that $i_{b}$ reaches $-I$ (Fig. 5c). In both the modes i) and iii), the commutation continues with a second subinterval where $i_{a}$ vanishes (mode i) and $i_{b}$ gets the required magnitude (mode iii); at the completion of this subinterval, denoted with \#2, commutation is completed and the current vector becomes equal to $\boldsymbol{I}_{1}$. Subinterval \#2 lasts $\theta_{c i}=\theta_{c l}-\theta_{i}$ in mode i) and $\theta_{c o}=\theta_{c h}-\theta_{o}$ in mode iii).

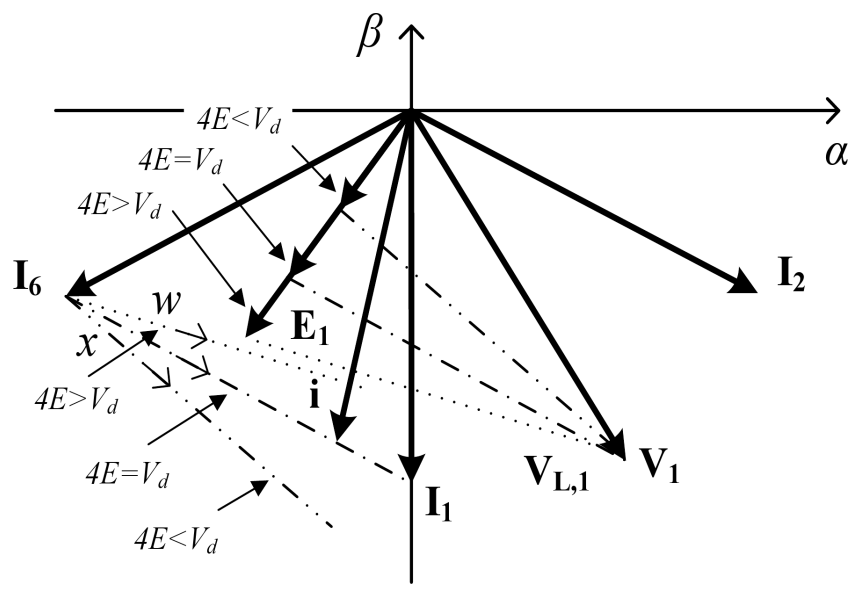

Fig. 6. Current vector trajectories during subinterval \#1

3.2. Current transients in low-speed zone. During subinterval \#1 the tip of the current vector moves along a line like $x$, as explained in the previous Subsection. At the end of the subinterval \#1, the current of phase $b$ reaches the required magnitude $-I$, i.e. it is $i_{b l}\left(\theta_{i}\right)=-I$, while the current of phase $a$ is still flowing.

During subinterval \#2, the drive control system regulates $i_{b}$ at $-I$ by applying the voltage $v_{b o}=V_{d}-E$ at the output $b$ of the VSI, while the other two outputs of the VSI are kept at the same voltages as before, i.e. it is $v_{a o}=V_{d}$, and $v_{c o}=V_{d}$, for $i_{a}$ to vanish. The vectors of the VSI output voltages and the voltage drops across the phase inductances are expressed as

$$
\begin{gathered}
\boldsymbol{V}_{2 \ell}=\left(\frac{1}{3}-j \frac{1}{\sqrt{3}}\right) E \\
\boldsymbol{V}_{L, 2 \ell}=\left(1+j \frac{1}{\sqrt{3}}\right) E .
\end{gathered}
$$

Both the vectors have a magnitude that depends on the speed and a slope that is independent of the speed. In particular, the slope of the vector in (17) is

$$
m_{2 \ell}=\frac{1}{\sqrt{3}}
$$

that, as shown in Fig. 7, is the same as the line $s$. Substitution of (17) into (11) and integration of (11) give the current vector in subinterval \#2

$$
\begin{gathered}
\boldsymbol{i}_{2 \ell}\left(\theta_{e}-\theta_{i}\right)=\left(1+j \frac{1}{\sqrt{3}}\right) \frac{E}{\Omega_{e} L}\left(\theta_{e}-\theta_{i}\right)+\boldsymbol{i}_{1 \ell}\left(\theta_{i}\right) \\
\theta_{e}>\theta_{i},
\end{gathered}
$$

where $\boldsymbol{i}_{1 \ell}\left(\theta_{i}\right)$ is the current vector at the end of subinterval $\# 1$. The angle $\theta_{i}$ can be obtained by calculating the current vector at $\theta_{e}=\theta_{i}$ by means of the first equation in (7), which becomes

$$
\mathbf{i}_{1 \ell}\left(\theta_{i}\right)=i_{a}\left(\theta_{i}\right)+j \frac{1}{\sqrt{3}}\left[-2 I+i_{a}\left(\theta_{i}\right)\right] .
$$

Then, by equating the real and imaginary parts of (20) to (15), also calculated at $\theta_{e}=\theta_{i}$, the values of $\theta_{i}$ and $i_{a}\left(\theta_{i}\right)$ can be obtained. In particular, $\theta_{i}$ results in

$$
\theta_{i}=\frac{3 \Omega_{e} L I}{2\left(V_{d}-E\right)} .
$$

Finally, substitution of (21) into (15) yields $\boldsymbol{i}_{1 \ell}\left(\theta_{i}\right)$.

The trajectory of the tip of the current vector in subinterval \#2, i.e. of $\boldsymbol{i}_{2 \ell}\left(\theta_{e}-\theta_{i}\right)$, is a straight line having the slope in (18). Since the current vector coincides with $\boldsymbol{I}_{1}$ at the end of subinterval \#2, and the slope of the vector $\boldsymbol{I}_{21}=\boldsymbol{I}_{2}-\boldsymbol{I}_{1}$ is the same as (18), the trajectory is represented by the line $s$ of Fig. 7 that passes through the tips of the vectors $\boldsymbol{I}_{1}$ and $\boldsymbol{I}_{2}$. Thus, it can be stated that subinterval \#1 ends when the line $x$ intersects the line $s$.

Subinterval \#2 finishes when the current vector reaches $\boldsymbol{I}_{1}$. This occurs when the current of the phase $a$ vanishes. Being $i_{a}$ equal to the real part of the current vector, the duration of subinterval \#2 can be obtained by equating to zero the real part of (19) calculated at $\theta_{e}=\theta_{c l}$. It is

$$
\theta_{c i} \equiv \theta_{c \ell}-\theta_{i}=\frac{\Omega_{e} L I}{2 E}\left(\frac{V_{d}-4 E}{V_{d}-E}\right)
$$

and, from (21) and (22), the duration of the commutation interval is

$$
\theta_{c \ell}=\frac{\Omega_{e} L I}{2 E} \equiv \frac{n_{p} L I}{2 k} .
$$

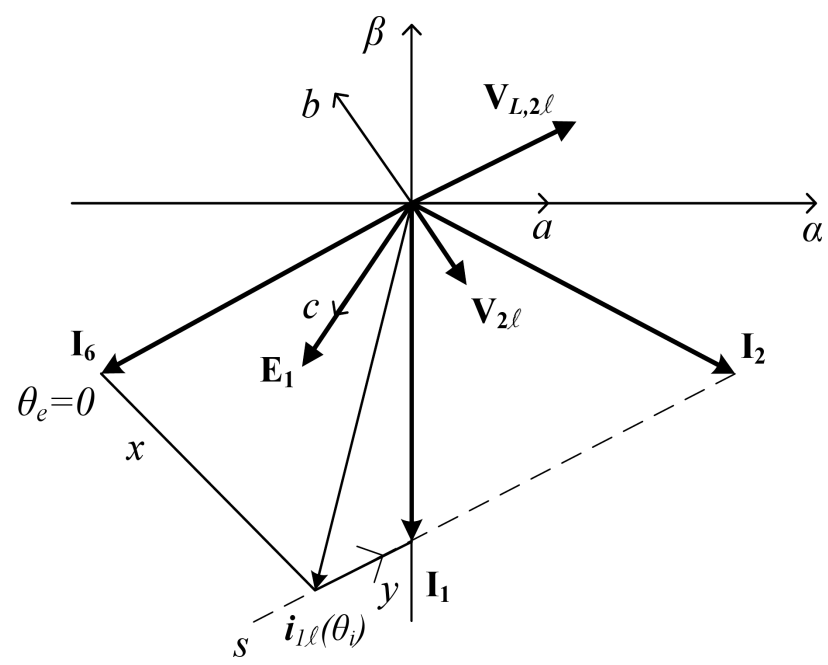

Fig. 7. Phase vectors during subinterval \#2 in low-speed zone

It is worth to note that the commutation interval does not depend on the motor speed and, furthermore, it coincides with the commutation interval as obtained for $4 E=V_{d}$.

The transients of the phase currents in the low-speed zone drawn in Fig. 5 (a) can be readily found by projecting the current vector on the axes $a, b$, and $c$ of Fig. 7 . 
3.3. Current transients in high-speed zone. During subinterval \#1 the tip of the current vector moves along a line like $w$, as explained in Subsec. 3.1). At the end of the subinterval $\# 1$, the current of phase $a$ vanishes, i.e. it is $i_{a h}\left(\theta_{o}\right)=0$, while the current of phase $b$ is still on the way to get the required magnitude. This means that at $\theta_{e}=\theta_{o}$ the real part of the current vector becomes zero and hence subinterval \#1 finishes when the line $w$ intersects the current vector $\boldsymbol{I}_{1}$.

The angle $\theta_{o}$ is found by equating to zero the real part of (15) calculated at $\theta_{e}=\theta_{o}$ and is equal to

$$
\theta_{o}=\frac{3 \Omega_{e} L I}{V_{d}+2 E}
$$

Substitution of $\theta_{e}=\theta_{o}$ in (15) results in only imaginary part and gives the current vector at the end of subinterval \#1; it is

$$
\boldsymbol{i}_{1 h}\left(\theta_{o}\right)=-j\left[4\left(\frac{V_{d}-E}{V_{d}+2 E}\right)\right] \frac{I}{\sqrt{3}} .
$$

As anticipated, the vector is aligned along $\boldsymbol{I}_{1}$ and has a magnitude lower than $2 I / \sqrt{3}$.

During subinterval \#2, the control system does not chop the VSI transistors $T_{6}$ and $T_{5}$ but keeps them ON to facilitate the incoming current to reach the required magnitude. Then only the legs $b$ and $c$ of the VSI conduct and the voltage of the neutral point of the motor with respect to $o$ is $V_{d} / 2$. Let us suppose that the leg $a$ is fictitiously chopped at the voltage of $v_{a o}=e_{a}+V_{d} / 2$ so as to maintain the zero current condition for the phase $a$. The other output voltages of the VSI are $v_{b o}=0$ and $v_{c o}=V_{d}$. Thus the vector of the output VSI voltages can be determined and results in

$$
\boldsymbol{V}_{2 h}=-\frac{2}{3} E-j \frac{1}{\sqrt{3}} V_{d}
$$

whilst the vector of the voltage drops across the phase inductances becomes

$$
\boldsymbol{V}_{L, 2 h}=-j \frac{1}{\sqrt{3}}\left(V_{d}-2 E\right) .
$$

The two voltage vectors in (26) and (27) have both the magnitude and the slope that depend on the motor speed.

From (11) and (27), the current vector is given by

$$
\boldsymbol{i}_{2 h}\left(\theta_{e}-\theta_{o}\right)=-j \frac{1}{\sqrt{3}}\left(\frac{V_{d}-2 E}{\Omega_{e} L}\right)\left(\theta_{e}-\theta_{o}\right)+\boldsymbol{i}_{1 h}\left(\theta_{o}\right) \text {. }
$$

Equations (25) and (28) point out that the tip of the current vector moves along the imaginary axis as shown in Fig. 8. Magnitude of the vector increases and then the tip continues to advance along $\boldsymbol{I}_{1}$ until it reaches the tip of $\boldsymbol{I}_{1}$ at $\theta_{e}=\theta_{c h}$. Duration of subinterval \#2 is obtained by equating (28) at $\boldsymbol{I}_{1}$. It is

$$
\theta_{c o} \equiv \theta_{c h}-\theta_{o}=\frac{2 \Omega_{e} L I\left(4 E-V_{d}\right)}{\left(V_{d}-2 E\right)\left(V_{d}+2 E\right)}
$$

and, from (24) and (29), the duration of the commutation interval is

$$
\theta_{c h}=\frac{\Omega_{e} L I}{V_{d}-2 E} \text {. }
$$

The transients of the phase currents in the high-speed zone drawn in Fig. 5 (c) can be readily found by projecting the current vector on the axes $a, b$, and $c$ of Fig. 8. As discussed in
[9], in high-speed zone $\boldsymbol{i}_{2 h}$ must equate $\boldsymbol{I}_{1}$ within $\theta_{e}=\pi / 3$ to get the required current reference at least at the end of the allowed commutation interval, i.e. of the supply interval.

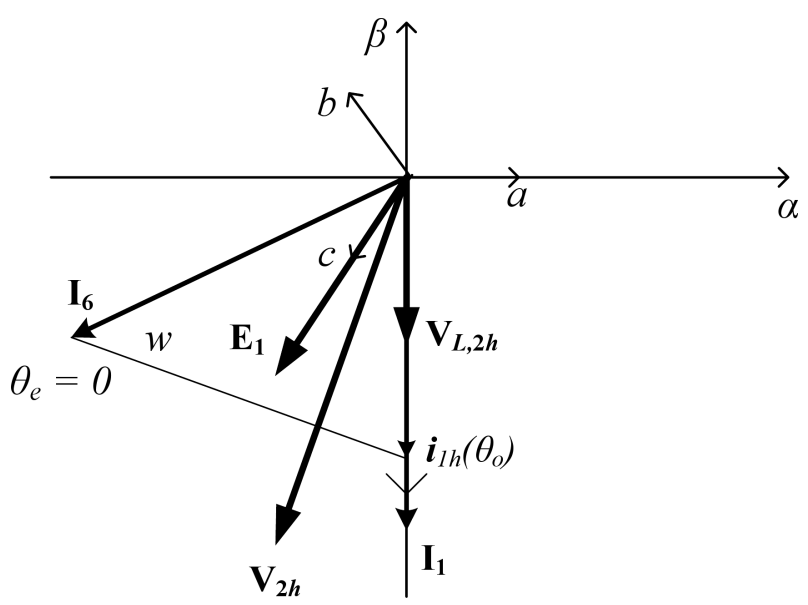

Fig. 8. Phase vectors during subinterval \#2 in high-speed zone

\section{Effects of current commutations on motor torque}

The instantaneous motor torque during current commutations can be still calculated by (5) and (9). For the current commutation occurring at the beginning of supply interval $S_{1}$, the instantaneous motor torque can be expressed as

$$
\tau\left(\theta_{e}\right)=\frac{3}{2 \Omega} \boldsymbol{E}_{1} \cdot \boldsymbol{i}\left(\theta_{e}\right) .
$$

By (31), the torque changes during the commutation interval. The change is proportional to the projection of $\left[\boldsymbol{i}\left(\theta_{e}\right)-\boldsymbol{I}_{1}\right]$ on $\boldsymbol{E}_{1}$ and, by accounting of the expressions of $\boldsymbol{i}\left(\theta_{e}\right)$, the change is a linear function of $\theta_{e}$.

In the low-speed zone, the projection of $\boldsymbol{i}\left(\theta_{e}\right)$ on $\boldsymbol{E}_{1}$ is greater than the projection of $\boldsymbol{I}_{1}$ and the instantaneous motor torque has a positive dip. The torque ripple, which is the absolute of the maximum excursion, is equal to

$$
T R_{\ell}=\left|\frac{3}{2}\left\{\frac{\boldsymbol{E}_{\mathbf{1}} \cdot\left[\boldsymbol{i}_{1 \ell}\left(\theta_{i}\right)-\boldsymbol{I}_{1}\right]}{\Omega}\right\}\right| .
$$

After some manipulations the following expression is obtained for (32)

$$
T R_{\ell}=\frac{n_{p} I}{\Omega_{e}}\left[\frac{\left(V_{d}-4 E\right) E}{V_{d}-E}\right] .
$$

In the high-speed zone, the projection of $\boldsymbol{i}\left(\theta_{e}\right)$ on $\boldsymbol{E}_{1}$ is lower than the projection of $\boldsymbol{I}_{1}$ and the instantaneous motor torque has a negative dip. The torque ripple is now equal to

$$
T R_{h}=\left|\frac{3}{2}\left\{\frac{\boldsymbol{E}_{\mathbf{1}} \cdot\left[\boldsymbol{i}_{1 h}\left(\theta_{o}\right)-\boldsymbol{I}_{1}\right]}{\Omega}\right\}\right| .
$$

After some manipulations the following expression is obtained for (34)

$$
T R_{h}=\left|\frac{2 n_{p} I}{\Omega_{e}}\left[\frac{\left(4 E-V_{d}\right) E}{V_{d}+2 E}\right]\right| .
$$


From Figs. 7 and 8, it can be easily realized that the torque ripple in both the low-speed and high-speed zones is proportional respectively to the maximum swing of the current of phase $c$.

Due to the torque dip, the motor torque, defined as the average value of the instantaneous motor torque over a supply period, changes with respect the expected value in (10), by increasing in the low-speed zone and decreasing in the highspeed zone. The terms in excess and in defect can be found by calculating the average value of the torque dip over the supply interval $\pi / 3$. It comes out

$$
\begin{gathered}
\Delta T_{\ell}=\frac{3}{\pi} \frac{1}{2} T R_{\ell} \theta_{c \ell}, \\
\Delta T_{h}=-\frac{3}{\pi} \frac{1}{2} T R_{h} \theta_{c h} .
\end{gathered}
$$

By substituting (23) and (33) into (36), and (30) and (35) into (37), the following expressions can be obtained for the two terms

$$
\begin{gathered}
\Delta T_{\ell}=\frac{3 n_{p} L I^{2}}{4 \pi}\left[\frac{V_{d}-4 E}{V_{d}-E}\right], \\
\Delta T_{h}=-\frac{3 n_{p} L I^{2}}{\pi}\left[\frac{\left(4 E-V_{d}\right) E}{\left(V_{d}+2 E\right)\left(V_{d}-2 E\right)}\right] .
\end{gathered}
$$

\section{Exemplification}

To demonstrate the potentialities of the vector approach in analyzing the operation of a PM BLDC drive, two control techniques of the VSI during the current commutations are considered, that have been proposed to eliminate the torque ripple produced by the commutations in the low-speed zone [13] and in the high-speed zone [14], respectively.

5.1. Low-speed zone. The control technique in [13] proposes to slow down reaching of the required magnitude from the incoming current $i_{b}$ by forcing it to have a magnitude complementary to that one of the outgoing current $i_{a}$, i.e. by taking constant the sum of the magnitudes of $i_{b}$ and $i_{a}$ and equal to the required current magnitude $-I$. To fulfill this condition, [13] chops the transistor $T_{6}$ of the incoming phase during the commutation interval with the following duty-cycle:

$$
\delta_{\ell}=\frac{4 E}{V_{d}} .
$$

The VSI output voltages are then: $v_{a o}=V_{d}, v_{b o}=\left(1-\delta_{l}\right) V_{d}$, $v_{c o}=V_{d}$. By (7) and (11), the vectors of the VSI output voltages and the voltage drops across the phase inductances become

$$
\begin{gathered}
\boldsymbol{V}_{1 \ell}=\left[\frac{1}{3}-j \frac{1}{\sqrt{3}}\right] 4 E, \\
\boldsymbol{V}_{L, 1 \ell}=\left[1-j \frac{1}{\sqrt{3}}\right] 2 E .
\end{gathered}
$$

Note that the voltage vector in (41) has the same direction as $\boldsymbol{V}_{1}$ in (12) and a magnitude scaled of $\delta_{l}$. As it can be recognized from Fig. 9, this control technique forces the voltage vector (42) to stay in parallel to the line joining $\boldsymbol{I}_{6}$ and $\boldsymbol{I}_{1}$ irrespectively from the motor speed, so that the current vector during the commutation moves along this line and its magnitude does not exhibit any positive swing. The commutation interval spans only subinterval \#1 as the incoming current reaches the required magnitude at the same time as the outgoing current vanishes, and the commutation angle is still given by (23). Clearly, the duty-cycle in (40) can be applied only for $4 E<V_{d}$, i.e. for $\delta_{l}<1$, and hence this technique is effective only in the low-speed zone.

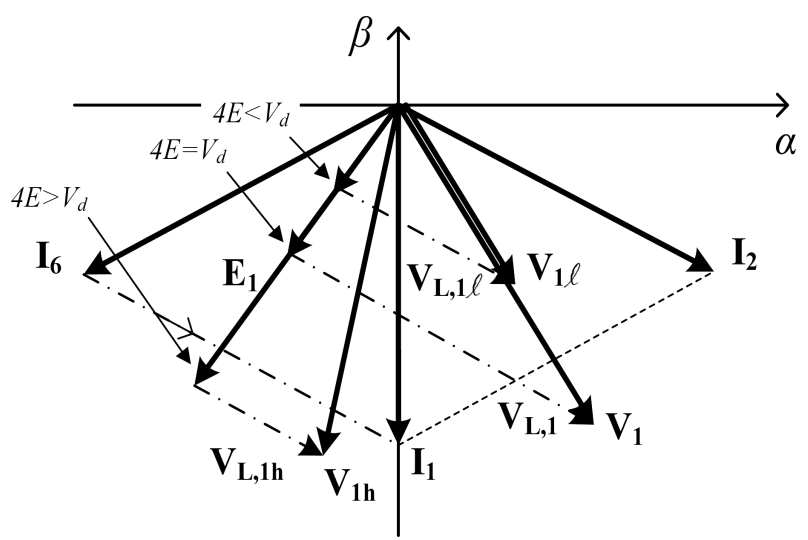

Fig. 9. PM BLDC control techniques to eliminate torque ripple

5.2. High-speed zone. The control technique in [14] proposes to slow down vanishing of the outgoing current $i_{a}$ by forcing it to have a magnitude complementary to that one of the incoming current $i_{b}$, i.e. by taking constant the sum of the magnitudes of $i_{b}$ and $i_{a}$ and equal to the required current magnitude $-I$. To fulfill this condition, [15] chops the transistor $T_{4}$ of the outgoing phase during the commutation interval with the following duty-cycle:

$$
\delta_{h}=\frac{4 E}{V_{d}}-1 .
$$

The VSI output voltages are then: $v_{a o}=\left(1-\delta_{h}\right) V_{d}, v_{b o}=0$, $v_{c o}=V_{d}$. By (7) and (11), the vectors of the VSI output voltages and the voltage drops across the phase inductances become

$$
\begin{gathered}
\boldsymbol{V}_{1 h}=\left(V_{d}-\frac{8}{3} E\right)-j \frac{1}{\sqrt{3}} V_{d}, \\
\boldsymbol{V}_{L, 1 h}=\left(1-j \frac{1}{\sqrt{3}}\right)\left(V_{d}-2 E\right) .
\end{gathered}
$$

Note that direction of the vector in (44) depends on the motor speed; in spite of this, the vector in (45) is parallel to the line joining $\boldsymbol{I}_{6}$ and $\boldsymbol{I}_{1}$ irrespectively from the motor speed, as shown in Fig. 9, so that the current vector during the commutation moves along this line and does not exhibit any swing. Therefore this control technique operates in a similar way that the technique in the low-speed zone but with the difference of chopping the output VSI voltage of the outgoing phase instead of the incoming one. As above, the commutation interval spans only subinterval \#1 as the incoming current reaches the required magnitude at the same time as the outgoing current vanishes. Here, instead, the commutation angle changes with the speed and is still given by (30). As an example, Fig. 10 
gives the trajectory on the $\alpha, \beta$ plane of the current vector obtained without and with the control technique during the current commutations at high speeds. The curves, obtained by simulation, clearly show the beneficial effect of the control.

The duty-cycle in (43) can be applied only for $4 E>V_{d}$ and up to $2 E=V_{d}$, i.e. for $\delta_{h}<1$, and hence this technique is effective only in the high-speed zone. Note that extension of (44)-(46) to the case of $2 E=V_{d}$ leads to the situation where the DC link voltage is not more able to inject any current into the motor phases as the voltage drop on the phase inductances is zero.

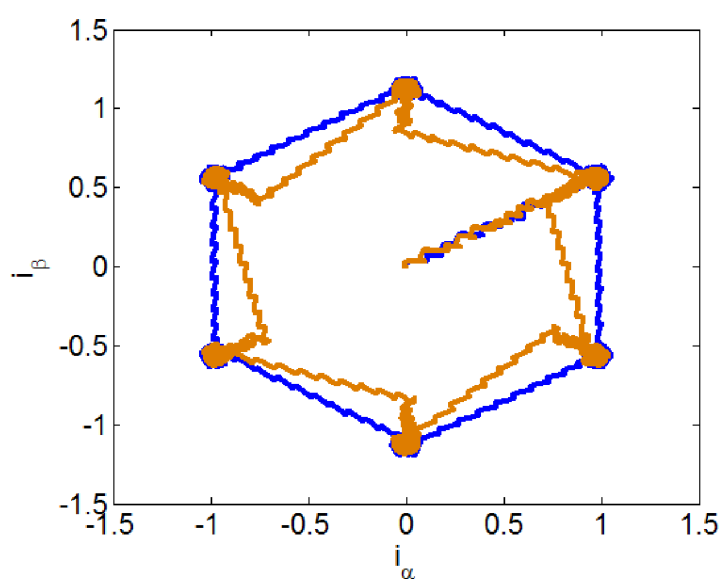

Fig. 10. Example of $\alpha, \beta$ plane trajectory of the current vector without (orange line) and with (blue line) VSI control during the current commutations at high speed

\section{Conclusions}

The current transients during the commutations of the PM BLDC drives have been analyzed in the stationary plane with the help of the vector representation of the drive variables. Vectors of the VSI output voltages and the voltage drops on the phase inductances have been calculated and utilized to get the current vector trajectories for different motor speeds as well as to find out the commutation intervals. The results of the analysis have been used to obtain the motor torque and the torque ripples due to the current commutations. Lastly, the vector approach has been applied to two control techniques of the PM BLDC drives with the end of explaining how they operate to eliminate the torque ripples due to the current commutations.

\section{REFERENCES}

[1] P. Pillay and R. Krishnan, "Application characteristics of permanent magnet synchronous and brushless DC motors for servo drives", IEEE Trans. on Industry Applications 27, 986-996 (1991).

[2] T.S. Low, T.H. Lee, K.J. Tseng, and K.S. Lock, "Servo performance of a BLDC drive with instantaneous torque control", IEEE Trans. on Industry Applications 28 (2), 455-462 (1992).

[3] A. Sathyan, M. Krishnamurthy, N. Milivojevic, and A. Emadi, "A low-cost digital control scheme for Brushless DC motor drives in domestic applications", Proc. IEEE Electric Machines and Drives Conf. (IEMDC) 1, 76-82 (2009).

[4] K.T. Chau, C.C. Chan, and C. Liu, "Overview of permanentmagnet brushless drives for electric and hybrid electric vehicles", IEEE Trans. on Industrial Electronics 55, 2246-2257 (2008).

[5] G. Buja and M. Bertoluzzo, "Development of electric propulsion systems for light electric vehicles", IEEE Trans. on Industrial Informatics 7 (3), 428-435 (2011).

[6] A.M. El-Refaie, "Motors/generators for traction/propulsion applications: a review", IEEE Vehicular Technology Magazine 8 (1), 90-99 (2013).

[7] T. Kenjo and S. Nagamore, Permanent-Magnet and Brushless DC Motors, Clarendon, Oxford, 1985.

[8] R. Krishnan, Permanent Magnet Synchronous Motors and Brushless DC Motor Drives, Taylor \& Francis, CRC Press, Boca Raton, 2010.

[9] T.J.E. Miller, Brushless Permanent-Magnet And Reluctance Motor Drives, Clarendon, Oxford, 1989.

[10] P. Pillay and K. Ramu, "Modeling, simulation and analysis of permanent magnet motor drives. Part II: the brushless DC motor drive", IEEE Trans. on Industry Applications 25 (2), 274-279 (1989).

[11] R. Carlson, M. Lajoie-Mazenc, and J.C. dos S.Fagundes, "Analysis of torque ripple due to phase commutation in brushless DC machine", IEEE Trans. on Industry Applications 25 (3), 632-638 (1992).

[12] G. Buja, M. Bertoluzzo, R. Keshri, and R. Menis, "Stationary plane based investigation of the behavior of PM BLDC Drives", Proc. IEEE Annual Conf. on Industrial Electronics (IECON) 1, 6630-6635 (2013).

[13] J. Fang, H. Li, and B. Han, "Torque ripple reduction in BLDC torque motor with nonideal back EMF", IEEE Trans. on Power Electronics 25 (11), 4630-4637 (2012).

[14] J.H. Song and I. Choy, "Commutation torque ripple reduction in brushless DC motor drives using a single DC current sensor", IEEE Trans. on Power Electronics 19, 312-319 (2004). 